\title{
Pleurotus djamor, un hongo con potencial aplicación biotecnológica para el neotrópico
}

\section{Pleurotus djamor, a mushroom with potential biotechological application for the Neotropic}

\author{
Dulce Salmones \\ Instituto de Ecología, A.C., Carretera antigua a Coatepec 351, El Haya, Xalapa, 91070, Veracruz, México. \\ Dulce Salmones, e-mail: dulce.salmones@inecol.mx
}

\section{RESUMEN}

Antecedentes: Pleurotus djamor es un hongo pantropical, cultivado comercialmente en algunos países asiáticos. Debido a sus características de crecimiento, ciclos de cultivo cortos y aceptable valor nutrimental, es una especie comestible adecuada para su propagación y consumo en las regiones cálidas del mundo.

Objetivo: El propósito de la presente revisión fue conocer los avances en la biología y cultivo de esta especie, con la finalidad de incrementar su potencial aprovechamiento en el neotrópico.

Método: Se hizo una revisión bibliográfica del tema, seleccionando información relevante sobre aspectos biológicos y biotecnológicos de la especie.

Resultados y conclusiones: Esta contribución revisa las características nutrimentales y funcionales de la especie, así como sus capacidades biosínteticas y biodegradativas en diversos procesos biotecnológicos, tales como producción de alimento, biotransformación de residuos orgánicos a través de la secreción de enzimas ligninocelulolíticas y su aplicación en procesos de biorremediación de suelos y agua, entre otros. P. djamor ha sido utilizado exitosamente en diferentes procesos biotecnológicos y representa un hongo comestible alternativo para el neotrópico, debido a las condiciones ambientales y disponibilidad de materiales lignocelulósicos existentes. La producción y consumo de esta especie podría mejorar las condiciones nutrimentales, ambientales y económicas de los pobladores de esta región.

Palabras clave: biodegradación, biosíntesis, hongos comestibles, setas, taxonomía.

\section{ABSTRACT}

Background: Pleurotus djamor is a pantropical fungus, cultivated commercially in some Asian countries. Due to its growth characteristics, short crop cycles and acceptable nutritional value, it is a mushroom suitable for its propagation and consumption in the warm regions of the world.

Objective: The purpose of this contribution was to review the advances in the biology and culture of this species, with the purpose of increasing its use in the Neotropic.

Method: A bibliographic review of the subject was made, selecting relevant information on biological and biotechnological aspects of this species.

Results and conclusions: This contribution summarize the nutrimental and functional characteristics of the species, as well as its biosynthetic and degradative capacity in various biotechnological processes, such as food production, biotransformation of organic residues by the action of ligninocellulolytic enzymes and their application in bioremediation processes of soils and water, among others. $P$. djamor has been used successfully in different biotechnological processes and represents an alternative species for the Neotropics, due to the environmental conditions and availability of lignocellulosic materials existing. The production and consumption of this mushroom could improve the nutrimental, environmental and economic conditions of the human population from this region.

Key words: biodegradation, biosynthesis, mushrooms, oyster mushroom, taxonomy. 


\section{INTRODUCCIÓN}

El género Pleurotus (Fr.) P. Kumm es cosmopolita, con especies que pueden crecer en altitudes tan variables como son, desde el nivel del mar hasta más de 3000 metros (Guzmán, 2000). Las especies del género se caracterizan por poseer un alto valor nutrimental, potencial nutraceútico y variadas aplicaciones biotecnológicas y ambientales (Cohen et al., 2002; Bordallo et al. 2002; Asaduzzaman Khan y Mousumi, 2012).

Guzmán (2000) consideró 50 especies válidas del género, aunque taxonómicamente es un grupo muy complejo ya que presenta especies con gran similitud morfológica. Genéticamente se han encontrado relaciones de intercompatibilidad y grupos de interesterilidad entre organismos pertenecientes o no a la misma especie, lo que ha complicado la sistemática del género (Salmones y Mata, 2017).

Pleurotus spp. es uno de los grupos más importantes de hongos comestibles cultivados comercialmente, con una producción estimada de $6.46 \times 10^{9} \mathrm{Kg}$ que los posiciona en el segundo lugar a nivel mundial (Royse et al., 2017). El 99\% de la producción de Pleurotus se concentra en el continente asiático, especialmente en China. En Latinoamérica, la producción comercial se genera mayoritariamente en Brasil, México, Colombia, Argentina y Guatemala (Royse y Sánchez, 2017). En México, el cultivo de hongos comestibles y funcionales es considerada una agroindustria promisoria y en expansión, que actualmente presenta varios retos para su consolidación, entre ellos, la adaptación de cepas a las condiciones ambientales, especialmente para las regiones tropicales y subtropicales (Martínez-Carrera et al., 2016). Las especies principalmente cultivadas en el país son P. pulmonarius y P. ostreatus, ambas preferentemente desarrolladas bajo condiciones templadas (Gaitán-Hernández y Salmones, 2008; Royse y Sánchez, 2017). Sin embargo, considerando el incremento de temperatura ambiental que gradualmente se está presentado debido a los efectos del cambio climático y, por otra parte, el que gran parte del territorio nacional presente condiciones ambientales cálidas (Villers-Ruiz y Trejo-Vázquez, 1998), es apremiante considerar la propagación comercial de otras especies adscritas al género que pudieran ser viables de producirse y comercializarse a corto plazo. Entre ellas, destaca la especie P. djamor, un hongo nativo capaz de desarrollarse a temperaturas cálidas, que se cultiva comercialmente en diversas regiones del mundo (Quimio, 1978; Rajarathnam et al., 1986; Miles y Chang, 2004) y que en Latinoamérica se ha cultivado principalmente con fines experimentales (Bononi et al., 1991; Rosado et al., 2002b; Valencia del Toro et al., 2003; Salmones et al., 1997, 2004, 2005; Vega et al., 2006; Ancona et al. 2007,) presentando características de cultivo y propiedades nutraceúticas y biodegradativas de interés para los productores regionales (Figura 1). En esta revisión, se presentan las habilidades biosintéticas y biodegradativas de esta especie tropical, destacando su potencial aprovechamiento biotecnológico para el neotrópico.

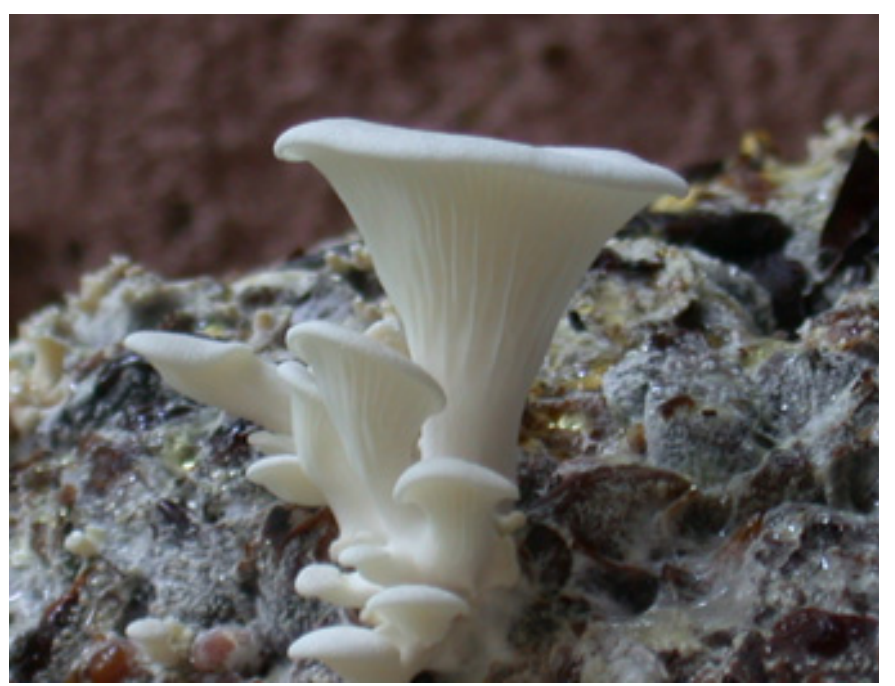

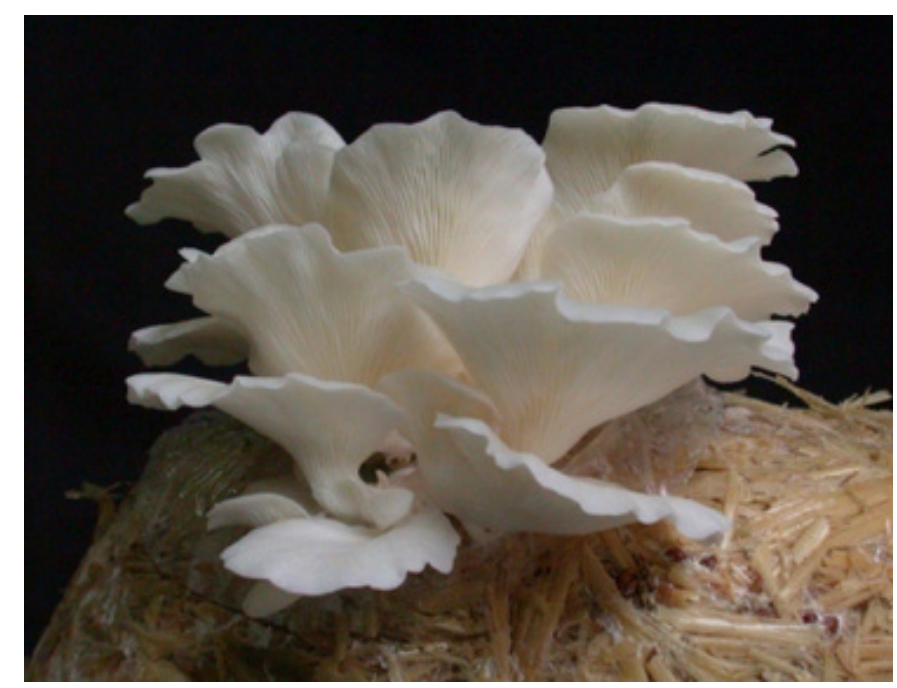

Figura 1. Basidiomas de P. djamor cultivados en paja de cebada (izquierda) y pulpa de café (derecha). 


\section{SISTEMÁTICA Y CARACTERÍSTICAS MORFOLÓGICAS}

Pleurotus djamor (Rumph.: Fr.) Boedijn fue originalmente descrito por Fries en 1821, como Agaricus djamor. Posteriormente Boedijn, en 1959, lo transfirió al género Pleurotus de la familia Pleurotaceae (Nicholl y Petersen, 2000).

De acuerdo con Pegler (1977, 1983, 1986), Guzmán (2000) y Nicholl y Petersen (2000), P. djamor es la especie pantropical más importante y abundante del género Pleurotus. Guzmán (2000), consideró que $P$. djamor es sinónimo de $P$. flabellatus, $P$. eous y $P$. salmoneostramineus. En el continente americano se le ha encontrado creciendo silvestre en Argentina, Bolivia, Brasil, Colombia, Costa Rica, Cuba, Guadalupe, Guatemala, Martinica, México, Panamá, Puerto Rico y Venezuela (Guzmán et al., 1993; Legon 1999; Putzke, 2002; Morales et al., 2003; Lechner y Wrigth, 2004; Guzmán y Piepenbring, 2011; Menolli et al., 2014; Melgarejo, 2015), aunque probablemente tiene una distribución más amplia.

Pegler (1986) describió a la especie con basidiomas de color blanco a blanquecino, aunque previamente Corner (1981) había observado una amplia variabilidad micromorfológica entre los especímenes estudiados provenientes de Malasia, determinando que esta especie podría clasificarse en seis variedades de acuerdo con su variabilidad en color, micromorfología y hábitat, además de presentar especímenes de clasificación intermedia entre las variedades establecidas. Guzmán et al., (1993) también notaron diferencias de coloración en los píleos de la especie estudiados, desde blanquecino a grisáceo o rosáceo, proponiendo una posible sinonimia con otras especies de Pleurotus, como P. salmoneostramineus y $P$. ostreatoroseus. Esta última especie fue estudiada por Murakami y Takemaru (1990), quienes observaron la formación de basidiocarpos blancos en la progenie de cepas de P. ostreatoroseus cultivadas en el laboratorio, por lo que consideraron que la variabilidad de color entre los especímenes silvestres y cultivados se debía a mutaciones controladas por genes recesivos. Paralelamente, Neda et al. (1988) descubrieron que los especímenes identificados como P. salmoneostramineus eran compatibles con aquellos registrados como $P$. djamor, concluyendo que las diferencias de color entre los basidiomas y basidiosporas no eran un factor determinante para separar a los especímenes en dos especies; coincidiendo con
Petersen y Hughes (1993) quienes encontraron que P. ostreatoroseus y $P$. salmoneostramineus eran genéticamente compatibles. Estudios posteriores mostraron compatibilidad entre las formas rosas y blancas (Petersen y Ridley, 1996, Nicholl y Petersen, 2000, Gaitán-Hernández y Salmones, 1999; Salmones et al., 2004), por lo que Guzmán et al. (1995) describieron tres variedades ecológicas en la especie: P. djamor (Fr.) Boedijn var. djamor, caracterizada por desarrollar primordios y basidiomas blanquecinos; P. djamor var. roseus Corner, conformada por individuos que presentan primordios de color rosa y/o naranja que disminuyen notoriamente su coloración en estado adulto y P. djamor var. salmoneostramineus (L. Vass) Guzmán, que se distingue por presentar primordios y basidiomas adultos de color rosa y/o naranja (Figura 2). Posteriormente, Lechnner et al. (2004) incorporaron la variedad cyathiformis Corner, caracterizada por representar basidiomas blanquecinos con manchas oscuras sobre el píleo.

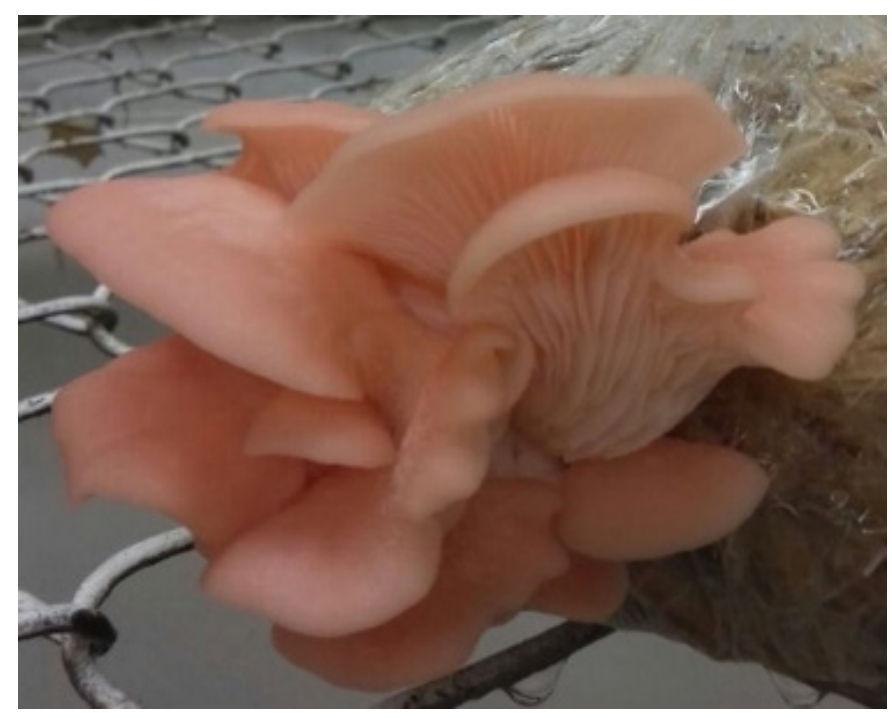

Figura 2. Pleurotus djamor var. salmoneostramineus creciendo en paja de cebada.

Con la incorporación de técnicas moleculares y bioquímicas en los estudios sistemáticos del género Pleurotus, la ubicación taxonómica de esta especie fue mejor definida. Zervakis y Labarere (1992) y Zervakis et al. (1994) después de analizar zimogramas de progenies homocarióticos y realizar pruebas enzimáticas con 11 especies de Pleurotus determinaron que $P$. 
djamor (citada como P. flabellatus) es una especie bien definida y separada de $P$. cornucopiae, $P$. cystidiosus, $P$. eryngii, $P$. ostreatus y $P$. pulmonarius. Por otra parte, Iracabal et. al. (1995) obtuvieron unidades de ADN ribosomal de P. cornucopiae con la finalidad de hibridizarlas con 11 taxa de Pleurotus, observando alta heterogeneidad entre los taxa y definiendo claramente tres especies: $P$. dryinus, $P$. eryngii y $P$. flabellatus $(P$. djamor), las cuales presentaron grandes distancias fenéticas con el resto de los taxones estudiados.

Es interesante destacar que la permanencia del color rosa en los basidiomas es una parte de la biología del hongo aún no concluyente, que ha generado conclusiones opuestas en los trabajos publicados, ya que mientras Cedano et al. (1993) citaron que la pérdida del color rosa en las fructificaciones cultivadas de una cepa mexicana de $P$. ostreatorroseus se debía a una alta intensidad lumínica en las áreas de cultivo, otros autores no encontraron correlación entre las variaciones de color rosa de los basidiomas y la intensidad de luz empleada durante el desarrollo de los mismos (Nicholl y Petersen, 2000). Probablemente esto se debe a que la permanencia del color depende, tanto de las características genéticas de los parentales como de las condiciones de cultivo y ambientales en que se desarrollan los esporomas, de igual manera a lo reportado para otras especies del género (Valencia del Toro y Leal-Lara, 1999; Guzmán, 2000).

Con referencia a su genética, presenta un patrón de sexualidad heterotálico tetrapolar (Chandrashekar et al., 1981), es decir sus talos o estructuras miceliales son autoestériles, por lo que para obtener descendencia se necesitan entrecruzar talos compatibles en dos pares de factores (A y B), localizados en dos cromosomas diferentes. James et al. (2004) reportó que la segregación de las secuencias de ADN en las regiones genómicas de la especie revelaba un par de genes homodominantes en el factor $\mathrm{A}$ y tres pares de receptores feromonales en el factor B, sugiriendo que las bases genéticas de $P$. djamor son similares a las de otras especies de hongos.

\section{VALOR NUTRIMENTAL}

Los esporomas de $P$. djamor presentan un aceptable contenido nutrimental. Estos hongos son especialmente ricos en carbohidratos, con 32.7 a 48.3 g por cada $100 \mathrm{~g}$ de hongo (base seca) y proteína cruda de 20.7 a 28 g, con valores recomendables de fibra cruda (9.1 a $22.4 \mathrm{~g}$ ) y cenizas (4.1 a $7.4 \mathrm{~g}$ ), baja cantidad de grasas (0.11 a $2.09 \mathrm{~g})$ y alta humedad (79.5 a 90\%) (Ancona et al., 2005; Mshandete y Cuff, 2007; Khan et al., 2013; Dharmarai et al., 2014; Selvakumar et al., 2015). La calidad nutrimental de la proteína de los hongos ha sido ampliamente estudiada, ya que una de las más importantes características biológicas de estos organismos son su capacidad de obtener el nitrógeno del sustrato de crecimiento y posteriormente sintetizarlo y transformarlo en aminoácidos. En este punto, los hongos del género Pleurotus son especialmente interesantes porque ellos pueden crecer en residuos lignocelulósicos con muy bajo contenido de nitrógeno, teniendo la capacidad de producir biomasa con mayor contenido de nitrógeno que el sustrato en el que están creciendo (Rajarathnam et al., 1998). Su calidad de proteína es alta, ya que está constituida principalmente por aminoácidos esenciales entre los que destacan la valina, isoleucina y fenilalanina, aunque también presentan valores aceptables de aminoácidos no esenciales como son, prolina, glutamato y aspartato (Ranzani y Sturion, 1998; Capetillo Leal et al., 2010). También contienen vitaminas, especialmente del complejo $\mathrm{B}\left(\mathrm{B}_{1}, \mathrm{~B}_{2}\right)$ y $\mathrm{D}$, además de los minerales $\mathrm{K}, \mathrm{P}, \mathrm{Mg}, \mathrm{Ca}, \mathrm{Na}, \mathrm{Zn}, \mathrm{Fe}$, entre los más frecuentes, por lo que los hongos se consideran una buena fuente de K y P (Guo et al., 2007; Rampinelli et al., 2010). Con base en su contenido nutrimental, Pleurotus djamor, y en general los hongos comestibles, son un alimento altamente recomendado para diversos tipos de dietas (Martínez-Carrera et al., 2016).

En cuanto a su palatabilidad, la especie se caracteriza por presentar un olor farináceo (harina húmeda) y cierta dureza y picor en sus fructificaciones frescas, aunque esta última característica es muy variable entre las cepas cultivadas (Ancona et al., 2005). Con referencia a su sabor, en el caso de los hongos éste proviene de compuestos volátiles derivados del metabolismo de sus ácidos grasos, predominantemente ácidos linoleicos y linolénicos, siendo el más importante el alcohol 1-octen-3-ol (Rajarathnam et al., 1998; Zawirska-Wojtasiak et al. 2009). Para la especie $P$. djamor (citada como P. flabellatus), se reportaron 16 compuestos que influyen en el sabor, siendo el 2-pentanona, 3-pentanona, butirato de metilo y 2-metil-3-pentanona, los mayormente encontrados por Rajarathnam et al. (1998); además del 3-octanol, 3-octanona y 1-octen-3-ol, reportados por Zawirska-Wojtasiak et al. (2009). 


\section{Propiedades medicinales}

Las propiedades medicinales de los hongos comestibles cultivados no sólo se han detectado en los esporomas, sino también, en el micelio e incluso en el medio de cultivo derivado del crecimiento micelial (Rosado et al., 2002a; Martínez-Carrera et al., 2016). Estas propiedades han sido ampliamente documentadas en las principales especies cultivadas y en el caso de $P$. djamor se han reportado compuestos bioactivos con propiedades anticancerígena, inmunomuladora, antibiótica (antimicrobianas, antiviral, antifúngica), antiparasitaria, antioxidante, antiinflamatoria, antidiabética, antilipidémica y hepaprotectora (Tabla 1$)$.

\section{CONDICIONES DE CULTIVO}

Al igual que otras especies del género, $P$. djamor presenta enzimas ligninocelulíticas que le permiten crecer en gran variedad de materiales agrícolas y forestales, especialmente bajo condiciones ambientales cálidas. Bano et al. (1979) y Geetha y Sivaprakasam (1993) encontraron que la especie (citada como $P$. flabellatus) es un hongo saprobio que crece en clima tropical a temperaturas óptimas entre 22 a $30{ }^{\circ} \mathrm{C}$. Las metodologías para la producción del hongo son similares a las empleadas para otras especies comerciales del género, como $P$. ostreatus y $P$. pulmonarius, con la diferencia de que su periodo de incubación es muy corto, incluso existen datos de obtención de la primera

Tabla 1. Propiedades medicinales encontradas en Pleurotus djamor

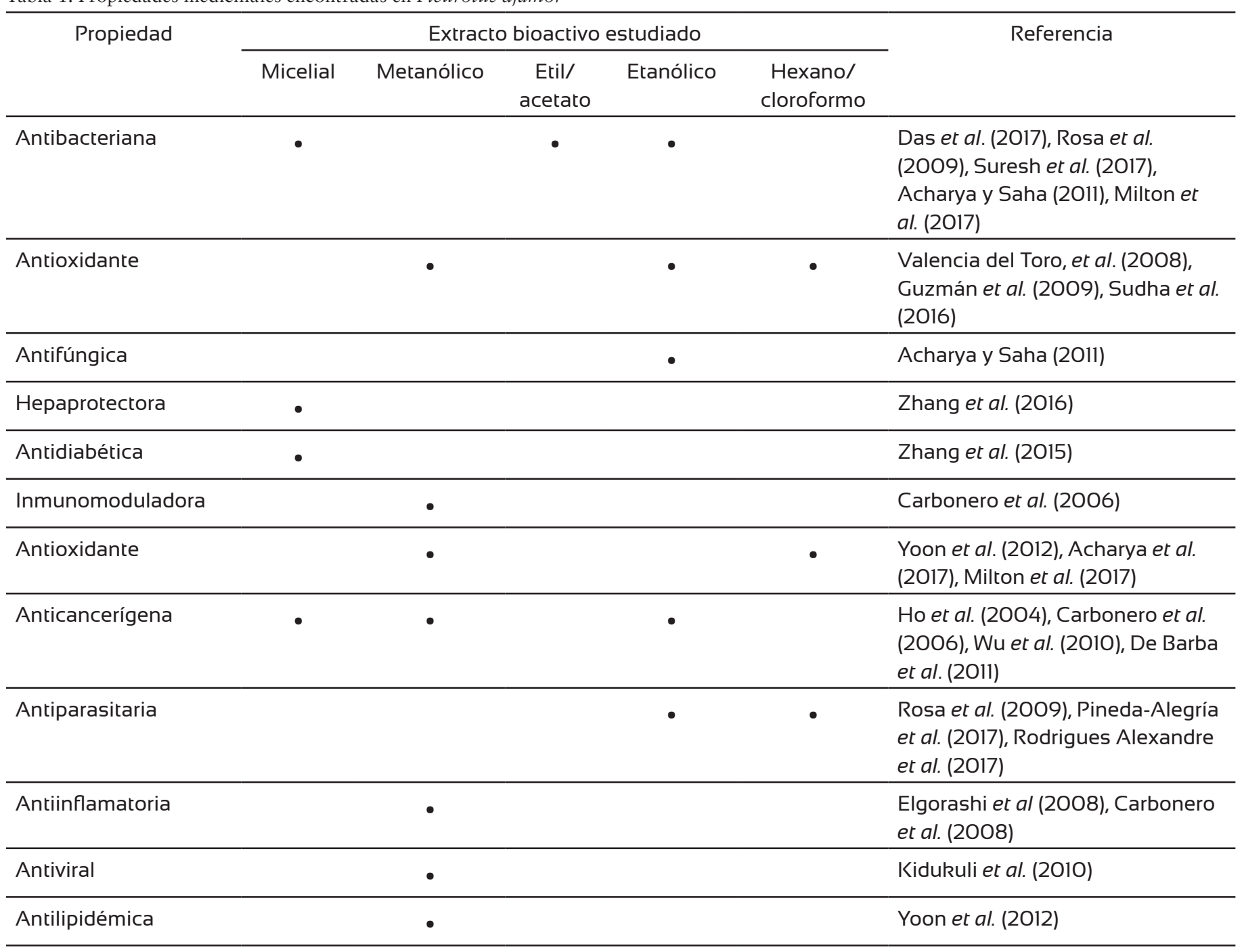


cosecha a partir de la segunda semana de incubación (Rajarathnam et al., 1986; Cetz et al., 2000), por lo que sus ciclos de cultivo son más cortos en comparación con las otras especies comerciales del género. A la fecha, una gran variedad de materiales lignocelulósicos ha sido utilizados para evaluar la productividad de la especie (Tabla 2).
A pesar de los avances significativos en la evaluación de sustratos para el cultivo de P. djamor, la disponibilidad de residuos agroindustriales en los trópicos es muy alta y se requiere intensificar la prospección de otros materiales orgánicos que permitan incrementar las eficiencias biológicas hasta ahora alcanzadas, con la finalidad de hacerlas competitivas con los

Tabla 2. Eficiencias biológicas de $P$. djamor alcanzadas en algunos subproductos agroindustriales

\begin{tabular}{|c|c|c|}
\hline Sustrato & EB (\%) & Referencia \\
\hline Algodón (Gossypium sp.) & & Funaki et al. (2010) \\
\hline Desechos & 92.5 & \\
\hline Arroz (Oryza sativa) & & Cayetano et al. (2007), Andrew et al. (2008), Vega y Franco (2013) \\
\hline Paja & 39.4-55.7 & \\
\hline Café (Coffea arabica) & 52.1-84.1, 40.2-61.8, & Hernández-lbarra et al. (1995), Salmones et al. (2005), Savoie et al. \\
\hline Pulpa & $63,64.4178 .3$ & (2007), Huerta et al. (2010) \\
\hline Calabaza (Cucurbita maxima) & & Cetz et al. (2000), Ancona et al. (2007) \\
\hline Rastrojo & $130,84-128$ & \\
\hline Caña de azúcar (Saccharum officinarum) & & Hasan et al. (2015), Selvakamur et al. (2015) \\
\hline Bagazo & $36.8,101.2$ & \\
\hline Cártamo (Carthamus tinctorius) & & Atila (2017) \\
\hline Paja & 77.8 & \\
\hline Coco (Cocos nucifera) & & Cayetano et al. (2007) \\
\hline Residuos del fruto & 35.3 & \\
\hline Frijol (Phaseolus vulgaris) & & Atila (2017) \\
\hline Rastrojo & 78.2 & \\
\hline Henequén (Agave fourcroydes) & & Ancona et al. (2007), Mshandete y Cuff (2008) \\
\hline Bagazo & $76-115$ & \\
\hline Residuos & $64-74$ & \\
\hline Lirio acuático (Eichhornia crassipes) & $53-84$ & Kivaisi et al. (2003) \\
\hline Maíz (Zea mays) & & Cayetano et al. (2007) Vega y Franco (2013); Menolli et al. (2010) \\
\hline rastrojo & $98,53.1$ & \\
\hline tuza & $16-27$ & \\
\hline Pajas de gramíneas & & Paswall et al. (2017); Salmones et al. $(1995,2004,2005)$; Savoie et \\
\hline avena (Avena sativa) & $51.9,56.5-123$ & al. (2007); Royse y Zaki (1991) Chaubey et al. (2010), Siddhant et \\
\hline cebada (Hordeum vulgare) & 53.6-114.4, & al. (2009) \\
\hline trigo (Triticum aestivum) & $30.5-40.4,60.2,72$ & \\
\hline Pastos (Pennisetum spp.) & & Bernardi et al. (2007); \\
\hline Pasto elefante (P. purpurem) & 132.6 & Gaitán-Hernández (1993) \\
\hline Zacate buffel (P. ciliare) & 54.1 & \\
\hline Plátano (Musa paradisiaca) & & Motato et al. (2006) \\
\hline hojas, pseudotallo y fruto & 24.1 & \\
\hline
\end{tabular}


rendimientos de las especies de Pleurotus cultivadas comercialmente. Generalmente, las fructificaciones de P. djamor se caracterizan por presentar cierta dureza en su estípite, lo que representa una desventaja de comercialización (ZawirskaWojtasiak et al. 2009); pero esto se compensa con la precocidad en la formación y madurez de sus esporomas. Además, esta capacidad del hongo para colonizar el sustrato en tiempos cortos de incubación, le da ventaja competitiva ante la presencia de mohos antagonistas, frecuentemente presentes en las diferentes etapas del proceso de cultivo (Salmones y Mata, 2015).

\section{CAPACIDAD BIODEGRADATIVA}

Los hongos del género Pleurotus están adaptados a crecer sobre una gran variedad de materiales lignocelulósicos, como las pajas de gramíneas, bagazos, residuos de la madera y de cultivos agrícolas (Tabla 2). Estos organismos están considerados como eficientes descomponedores de la lignocelulosa, principal polímero constituyente de los materiales lignocelulósicos, debido a que tienen la capacidad de sintetizar un amplio espectro de enzimas involucradas en los procesos de biodegradación y síntesis de compuestos participantes en las diferentes procesos vitales del organismo, como son: el metabolismo respiratorio, la construcción de biomasa fúngica y cuerpos fructíferos, la producción y descarga de las esporas y los mecanismos competitivos y defensivos, entre otros (Rajarathnam et al., 1998). Además, la degradación biológica de la lignocelulosa en compuestos de menor peso molecular y mayor digestibilidad, incluso para su aprovechamiento como alimento para consumo humano (las fructificaciones) y como forraje de animales (residuos sólidos con una digestibilidad mejorada), ha convertido el proceso del cultivo de especies comestibles de hongos en una atractiva alternativa del concepto de delignificación.

De acuerdo con los resultados publicados, las especies de Pleurotus presentan el tipo de la pudrición blanca selectiva, caracterizada por una degradación más rápida de la fracción lignina y una posterior despolimerización de la celulosa y hemicelulosa presentes (Leonowics et al., 2001; Mata et al., 2017). En lo general, las enzimas que degradan la celulosa y la hemicelulosa se encuentran asociadas a las etapas de producción de los basidiomas, mientras que las oxidasas aparecen durante las primeras etapas del desarrollo micelial (Salmones y Mata, 2002;
Mata et al., 2007). Sin embargo, este patrón de producción enzimática puede verse afectado por la composición química del sustrato de cultivo o por la presencia de microorganismos contaminantes (Mata et al., 2005; Salmones y Mata, 2015). Durante los últimos años, diversas investigaciones se han realizado para determinar el potencial de biodegradación de la lignocelulosa por especies comerciales de Pleurotus. Las enzimas ligninocelulolíticas producidas por Pleurotus spp. tienen un alto potencial de aplicación en las industrias papelera, química, textil y alimentaria, principalmente, aunque también tienen aplicación en procesos agrícolas y producción de forrajes para alimento animal (Rajarathnam et al., 1987, 1989; Cohen et al., 2002); y recientemente para la producción de bioenergéticos a partir de desechos agroindustriales (Sánchez-Cantú et al., 2017).

En cuanto a la especie $P$. djamor, los primeros estudios fueron realizados en la India por Rajarathnam et al. (1979), dichos autores observaron que al cultivar el hongo (citado como P. flabellatus) en paja de arroz, se presentaban pérdidas continuas en el contenido de celulosa, hemicelulosa, lignina, carbono y nitrógeno totales, desde el día de inoculación hasta la cosecha de los basidiomas, mientras que el contenido de azúcares libres, cenizas y relación $\mathrm{C} / \mathrm{N}$ se incrementaba. Los porcentajes de decremento cuantificados para la celulosa contenida variaron de 13.9 a $14 \%$, para las hemicelulosas de 6.6 a $7 \%$ y para la lignina de 1.5 a 4\%. El contenido de nitrógeno disminuyó entre 0.16 a $0.23 \%$. Estos autores concluyen que la progresiva degradación de la celulosa y hemicelulosa estaba relacionada con las actividades enzimáticas de las celulasas y hemicelulasas detectadas durante diferentes etapas del crecimiento del hongo. Kivaisi et al. (2003) citan una pérdida de fibra entre 31 al $40 \%$ al final del ciclo de cultivo, decreciendo la fracción de celulosa entre 35 a $48 \%$.

Rolz et al. (1986) cultivaron P. djamor en pasto de limón, determinando pérdidas en el sustrato residual de $42.6 \%$ de lignina, $32.35 \%$ de hemicelulosa y $22.58 \%$ de celulosa. En el mismo estudio, el hongo también fue cultivado en bagazo de citronela (Cymbopogon spp.) y para este sustrato los porcentajes de lignina, hemicelulosa y celulosa cuantificados fueron de $30,44.96$ y $36.57 \%$, respectivamente. Por otro lado, Capelari y Zadrazil (1997) cultivaron una cepa de P. ostratorosesus en paja, determinando pérdidas mayores al 50\% del contenido de 
liginina, después de 60 días de cultivo, especialmente cuando las temperaturas de incubación se mantuvieron entre $25^{\circ} \mathrm{C}$ a $30^{\circ} \mathrm{C}$.

En contraste, Okano et al. (2006) encontraron poca capacidad de digestibilidad de $P$. salmonostramineus en bagazo de caña, después de 12 semanas de incubación, por lo que concluyen que la especie presenta baja digestibilidad en cultivos in vitro, en comparación con otras especies cultivadas comercialmente como Lentinula edodes.

\section{BIORREMEDIACIÓN}

En la naturaleza los principales compuestos xenobióticos contaminantes del ambiente y susceptibles de eliminarse con hongos macroscópicos son: colorantes, aromáticos, hidrocarburos aromáticos, pesticidas y clorofenoles, por lo que los hongos lignocelulósicos han sido frecuentemente estudiados para determinar su capacidad de biorremediación (Cohen et al., 2002). Diversas cepas de Pleurotus comerciales han sido probadas con resultados prometedores para la producción de enzimas y la degradación de compuestos recalcitrantes (Mata et al., 2017).

Upadhyay y Hofrichter (1993) observaron que una cepa de P. djamor (citada como P. flabellatus) logró metabolizar 67 $\mathrm{mgL}^{-1}$ de fenol en 10 días, produciendo una pigmentación café sobre el medio de cultivo. Estudios preliminares habían indicado que la incorporación de fenol (hasta $1600 \mathrm{mgL}^{-1}$ ) durante la hidratación de las pajas, favoreció el crecimiento micelial de otras especies de Pleurotus e inhibió el crecimiento de organismos antagonistas, como Stachybotrys sp. y Coprinus sp.

Sánchez et al. (2007) lograron cultivar P. djamor en medio de cultivo sólido conteniendo endosulfán, un insecticida organoclorado utilizado en México para control de plagas de diversos cultivos de importancia económico; mientras que Ferrera Cerrato et al. (2007) cultivaron cepas de la especie in vitro utilizando petróleo crudo como fuente de carbono. Por otra parte, Selvakumar et al. (2007), mostraron que esta especie puede crecer en extractos de pino conteniendo una concentración de taninos crudos de hasta 10000 ppm, sin observar inhibición alguna en el desarrollo de los micelios.

Chan-Cupul et al. (2014) evaluaron el efecto de diferentes concentraciones de atrazina sobre el crecimiento micelial y la actividad enzimática de ocho macrohongos ligninolíticos, siendo la especie $P$. djamor más tolerante a la atrazina $\left(\mathrm{CE}_{50}=\right.$
$2281 \mathrm{mg} / \mathrm{l})$, aunque sus actividades enzimáticas no fueron altas, con respecto a las otras especies estudiadas.

Por otra parte, la industria textil es uno de los sectores más contaminantes ya que se estima que del 10 al 25\% de los colorantes aplicados para el teñido de telas, se vierten en efluentes de agua dulce, estando principalmente constituidos por compuestos aromáticos (antraquinonas y trifenilmetano) o del grupo azo (azobencenos), además de numerosos compuestos orgánicos tóxicos y recalcitrantes que afectan el ecosistema acuático; por lo que es necesario la remoción de los colorantes antes de su descarga en los cuerpos de agua, ya que actualmente es considerado uno de los principales problemas de contaminación ambiental (Thakur y Chauhan, 2018).

Estos compuestos son estables a la acción degradadora por la microbiota natural, porque presentan gran similitud química con algunos otros compuestos utilizados en los procesos vitales celulares. Para disminuir ese impacto negativo, existe la alternativa de usar las enzimas extracelulares ligninolíticas producidas por los hongos. Kalmis et al. (2007) realizaron un ensayo para determinar la capacidad de decoloración de diversas especies de Pleurotus, entre ellas P. djamor cultivadas in vitro. Los resultados del estudio muestran que la especies de Pleurotus tuvieron la capacidad de decolorar totalmente el azul brillante de Remazol R (antraquinona) y el azul brillante de Levafix, en concentraciones mayores a $20 \mathrm{mgL}^{-1}$, mientras que otros colorantes, como el negro de Indanthren RB fueron parcialmente decolorizados. De acuerdo con dichos autores, las especies de Pleurotus cultivadas pudieron crecer adecuadamente en concentraciones de colorantes superiores a $200 \mathrm{mgL}^{-1}$. En un estudio posterior realizado por los mismos autores, se determinó que $P$. djamor también puede decolorizar el negro benazol ZN, a partir de cultivos iniciales conteniendo de 500 a $1000 \mathrm{mgL}^{-1}$ del colorante (Kalmis et al., 2008). Mientras que Nilsson et al. (2006) cultivaron la especie con la finalidad de determinar su capacidad de decoloración en desechos de colorantes textiles, mostrando que $P$. djamor puede degradar hasta el $67 \%$ de los compuestos en cultivos realizados en reactores continuos tipo Batch.

Arboleda et al. (2008) experimentaron con 19 cepas de diferentes especies de hongos de pudrición blanca provenientes de un bosque tropical de Colombia, evaluando su capacidad para decolorar los compuestos Azure B y azul de Coomassie en 
medio sólido. P. djamor logró disminuir la decoloración de los compuestos en medio líquido, aunque su capacidad de producción de enzimas ligninolíticas, específicamente lacasa, ligninasa y peroxidasa dependiente de $\mathrm{Mn}$, fue menor con respecto a las otras especies estudiadas. Tanto las cepas rosadas (consideradas como P. ostreatoroseus) como las blancas (citadas P. flabellatus) mostraron capacidad de crecimiento micelial y actividad de decoloración más fuerte que $P$. chrsosporium, especialmente cuando fueron cultivadas en medio sólido y suelo contaminado como organoclorinados, incluso logrando decolorar el azul brillante de Remazol.

Machado et al. (2005), realizaron un estudio para evaluar la actividad ligninolítica de 125 basidiomicetos aislados de ecosistemas tropicales. Entre las especies evaluadas en agar con extracto de malta y diversos colorantes, $P$. djamor presentó alta capacidad de decolorar el azul brillante Remazol R en cultivos de agar con extracto de malta.

Santos et al. (2002) utilizaron una cepa rosada (citada como $P$. ostreatoroseus) para tratar efluentes de una planta de papel kraft blanco, logrando inicialmente una remoción de $18.6 \%$ de color y un $11.6 \%$ de fenoles totales. En un segundo experimento y después de añadir una fuente extra de carbono (glucosa), la máxima remoción de color, fenoles totales y lignina/clorolignina fueron de 19.4, 9.4 y 44.5\%. Los mejores resultados del estudio se alcanzaron utilizando una suspensión de $20 \%$ de masa micelial con el efluente, en agitación, ya que al tercer día los porcentajes de remoción de color, fenoles totales y lignina/clorolignina fueron de $84.4,82.1$ y $72.4 \%$, respectivamente.

Por otra parte, es importante considerar que, debido a la capacidad degradativa que caracteriza a los hongos, parte de elementos y/o compuestos químicos pueden concentrarse en el micelio y las fructificaciones del hongo. Brunnert y Zadrazil (1983) reportaron que una cepa de $P$. djamor, citada como $P$. flabellatus, logró incorporar a sus cuerpos fructíferos el $75 \%$ de cadmio y el $38.5 \%$ de mercurio depositado en los cultivos, destacando que, a diferencia de otras especies de hongos, P. flabellatus almacenó más cadmio que mercurio en sus fructificaciones (relación Cd/Hg 0.65). Mientras que Maihara et al. (2008), reportaron que una cepa cultivada de la especie, citada como $P$. salmoneostramineus, presentó $0.229 \mathrm{~m} / \mathrm{kg}$ de arsénico en los esporomas cultivados, aunque este valor es más bajo que los niveles máximos recomendados para el consumo de alimentos.

De los resultados obtenidos hasta ahora con $P$. djamor, se puede concluir que la especie presenta un gran potencial como degradadora de compuestos recalcitrantes, debido a su capacidad de secretar diversas enzimas ligninocelulolíticas durante su ciclo de vida, tanto en la etapa de colonización del sustrato, como durante la formación y desarrollo de las fructificaciones (Salmones et al., 2005). Sin embargo, la mayoría de los estudios realizados han sido a nivel de laboratorio, por lo que será necesario verificar si la capacidad biodegradativa del hongo se mantiene a nivel planta piloto, y preferentemente, bajo las condiciones ambientales propias del trópico. Escalar el proceso enfrenta otros retos metodológicos, tanto en el manejo del sustrato como en la producción de biomasa fúngica, pero finalmente esto podría favorecer los costos del proceso, lo que repercutiría en el interés de utilizar esta especie hongo en diversos procesos de biorremediación.

\section{CONCLUSIONES}

Pleurotus djamor es un hongo comestible cultivado exitosamente en algunos países asiáticos. El conocimiento actual que se tiene de su biología, fisiología, enzimología y biología molecular es considerable y ha permitido desarrollar su cultivo con la finalidad de obtener alimento para consumo humano con aceptable valor nutrimental, además de explorar las posibilidades de aprovechar sus propiedades biosintéticas y degradativas en otros procesos biotecnológicos. Debido a las condiciones climáticas y a la disponibilidad de materiales lignocelulósicos disponibles en el neotrópico, el cultivo de esta especie comestible resulta viable económica y ambientalmente. La duración del proceso puede ser menor a los ciclos de cultivo de otras especies comerciales de Pleurotus y las similitudes morfológicas con éstas, favorecería una rápida aceptación en el mercado regional. Cosechar todo el año resulta altamente ventajoso para los productores, por lo que la propuesta de contar con cepas para los meses calurosos deberá ser bien recibida por el sector productivo. Además, hay un interés creciente entre la población de consumir alimentos que contengan compuestos bioactivos y los hongos son los organismos más prometedores para descubrir nuevos fármacos a partir de sus metabolitos primarios y secundarios. Existen aún 
interrogantes sobre la acción del sistema enzimático de la especie y su potencial aprovechamiento en procesos de remediación de suelos y agua, así como en la biodegradación de plásticos y sus derivados, pero con las nuevas herramientas moleculares es muy probable que el avance en la interpretación del metabolismo del hongo y sus procesos biológicos se acelere. En conclusión, la producción y consumo de esta especie, representa un beneficio para la población.

\section{AGRADECIMIENTOS}

La autora agradece a las autoridades del Instituto de Ecología, A. C., las facilidades técnicas y el soporte financiero otorgado para realizar sus investigaciones. A Gerardo Mata se le agradece la revisión crítica del manuscrito, así como el préstamo de las fotografías que ilustran la figura 1, al Dr. Homero Lumbreras se le agradece el préstamo de la fotografía de la Figura 2.

\section{REFERENCIAS}

Acharya, S., A.K. Saha, 2011. Antimicrobial activity of Pleurotus djamor. Journal of Mycopathological Research 49(2): 329-332.

Acharya, K., S. Khatua, S. Ray, 2017. Quality assessment and antioxidant study of Pleurotus djamor (Rumph. ex Fr.) Boedijn. Journal of Applied Pharmaceutical Science 7 (6): 105-110.

Ancona, L., S. Medina, G. Cetz, 2005. Preferencia en el consumo de Pleurotus djamor en Baca, Yucatán, México. Revista Mexicana de Micología 20: 39-44.

Ancona, L., G. Cetz Zapata, R. Belmar Casso, C. Sandoval Castro, 2007. Cultivo de Pleurotus djamor y P. ostreatus en Yucatán. In: Sánchez Vázquez, J.E., D. Martínez Carrera, G. Mata, H. Leal Lara (eds.), El cultivo de setas Pleurotus spp. en México. El Colegio de la Frontera Sur. Pp. 131-142.

Andrew, S.M., S.M.S. Maliondo, P.K. Munishi, H. Msita, 2008. Yield of edible Pleurotus mushrooms grown on rice straw with and without chicken manure supplementation in Morogoro, Tanzania. Tanzania Journal of Forestry and Nature Conservation 77: 46-53.

Arboleda, C., A. Mejía, A.E. Franco-Molano, G.A. Jiménez, M. J. Pennicnckx, 2008. Autochthonous white rot fungi from the tropical forest of Colombia for dye decolourisation and ligninolytic enzymes production. Sydowia 60(2): 165-180.

Asaduzzaman Khan, M.D., T. Mousumi, 2012. Nutritional and medicinal importance of Pleurotus mushrooms: an overview. Food Review International 28(3): 313-329.

Atila, F., 2017. Evaluation of suitability of various agro-wastes for productivity of Pleurotus djamor, Pleurotus citrinopileatus and Pleurotus eryngii mushrooms. Journal of Experimental Agriculture International 17(5): 1-11.

Bano Z., S. Rajarathnam, N. Nagrajan, 1979. Some aspects on the cultivation of Pleurotus flabellatus in India. Mushroom Science 10 (2): 597-608.

Bernardi, E., L.P. Donini, E. Minotto, J.S. do Nascimento, 2007. Cultivation of three Pleurotus (Jacq.: Fr.) P. Kumm. species on pasteurized elephant grass (Pennisetum purpureum) substrate. International Journal of Medicinal Mushrooms 9(3-4): 373-378.
Bononi, V.L.R, R. Maziero, M. Capelari, 1991. Pleurotus ostreatoroseus cultivation in Brazil. Mushroom Science 12 (2): 531-532.

Brunnert H., F. Zadrazil, 1983. The translocation of mercury and cadmium into the fruiting bodies of six higher fungi. Applied Microbiology and Biotechnology 17(6): 358-364.

Bordallo J.J., L.V. Lopez-Llorca, H.B. Jansson, J. Salinas, L. Persmark , L. Asensio, 2002. Effects of egg-parasitic and nematode-trapping fungi on plant roots. New Phytologist 154: 491-499.

Capetillo Leal, C., L. Ancona Méndez, C. Sandoval Castro, G. Cetz Zapata, 2010. Chemical composition and amino acid profile of Pleurotus djamor and Pleurotus ostreatus cultivated in Mexico. Acta Alimentaria 39(3): 249-255.

Carbonero, E.R., A.H.P. Gracher, M.C.C. Rosa, G. Torri, G.L. Sassaki, P.A.J. Gorin, M. Iacomini, 2008. Unusual partially 3-O-methylated alpha-galactan from mushrooms of the genus Pleurotus. Phytochemistry 69(1): 252-257.

Carbonero, E.R., A.H.P. Gracher, F.R. Smiderle, F.R. Rosado, G.L. Sassaki, P.A.J. Gorin, M. Iacomini, 2006. A beta-glucan from the fruit bodies of edible mushrooms Pleurotus eryngii and Pleurotus ostreatoroseus. Carbohydrate Polymers 66(2): 252-257.

Cayetano Catarino, M., G. Mata, T. Bernabé González, 2007. Cultivo de Pleurotus ostreatus y $P$. djamor sobre dos subproductos agrícolas en Guerrero. In: Sánchez Vázquez, J.E., D. Martínez Carrera, G. Mata, H. Leal Lara (eds.). El cultivo de setas Pleurotus spp. en México. El Colegio de la Frontera Sur. Tapachula, pp. 113-122.

Cedano M., M. Martinez, C. Soto Velazco C., L. Guzman Davalos, 1993. Pleurotus ostreatoroseus (Basidiomycotina, Agaricales) in Mexico and its growth in agroindustrial wastes. Cryptogamic Botany 3(4): 297-302.

Cetz, G., L. Ancona, R. Belmar, 2000. Cultivo de Pleurotus djamor en rastrojo de calabaza. Revista Mexicana de Micología 16:41-43.

Chan-Cupul, W., G. Heredia Abarca, R. Rodríguez Vázquez, D. Salmones, R. Gaitán-Hernández, E. Alarcón Gutiérrez, 2014. Response of ligninolytic macrofungi to the herbicide atrazine: dose-response. Revista Argentina de Microbiología 46(4): 348 357.

Chandrashekar, T.R., Z. Bano, S. Rajarathnam, 1981. Incompatibility and growth in Pleurotus flabellatus; edible mushroom, mating system, commercial potential. Transactions British of Mycological Society 77(3):491-495.

Chaubey, A., P. Dechariya, D. Vyas, 2010. Suitability of various wastes for the cultivation of Pleurotus djamor. Mushroom Research 19(1): 36-39.

Cohen, R., L. Persky, Y. Hadar, 2002. Biotechnological applications and potential of wood-degrading mushrooms of the genus Pleurotus. Applied Microbiology and Biotechnology 58(5): 582-594.

Corner, E.J.H., 1981. The agaric genera Lentinus, Panus and Pleurotus. Beih. Nova Hedwigia 69: 1-169.

Das, A.R., A.K. Saha, S.R. Joshi, P. Das, 2017. Wild edible macrofungi consumed by ethnic tribes of Tripura in Northeast India with special reference to antibacterial activity of Pleurotus djamor (Rumph. ex Fr.) Boedijn. International Food Research Journal 24(2): 834-838.

De Barba, F.F.M., M.L.L. Silveira, B.U. Piloni, S.A. Furlan, M.S.L. Pinho, 2011. Influence of Pleurotus djamor bioactive substances on the survival time of mice inoculated with Sarcoma 180. International Journal of Pharmacy 7: 478-484.

Dharmarai, K., R. Kubera, R. Mahalakshmi, 2014. Comparison of nutrient contents and antimicrobial properties of Pleurotus djamor, Agaricus bisporus and Ganoderma tsugae. International Journal Current of Microbiology and Applied Sciences 3(6): 518-526.

Elgorashi, E. E., N. Maekawa, H. Satoh, 2008. In vitro anti-inflammatory activity of selected Japanese higher Basidiomycetes mush- 
rooms. International Journal of Medicinal Mushrooms 10(1): 49-53.

Ferrera Cerrato, R., M.E. Lara Hernández, J.E. Sánchez Vázquez, 2007. El género Pleurotus y su capacidad de crecer en medios de cultivo y suelo con diferentes concentraciones de petróleo. In: Sánchez Vázquez, J.E., D. Martínez Carrera, G. Mata, H. Leal Lara (eds.). El cultivo de setas Pleurotus spp. en México. El Colegio de la Frontera Sur. Tapachula. Pp.185-190.

Funaki dos Reis, M., F. Ducca, D.M. Ferdinandi, P. de Costa Zonetti, F.R. Rosado, 2010. Análise de substratos alternativos para o cultivo de Pleurotus ostreatoroseus e Pleurotus florida. Revista em Agronegocio e Meio Ambiente 3(2):79-91.

Gaitán-Hernández, R., D. Salmones, 1999. Análisis de la producción de cepas de Pleurotus djamor. Revista Mexicana de Micología 15: $115-118$.

Gaitán-Hernández, R., 1993. Cultivo de Pleurotus djamor en zacate buffel, viruta de Encino y bagazo de henequén. Reporte Científico 13 (especial): 111-115.

Gaitán-Hernández, R., D. Salmones D., 2008. Obtaining and characterizing Pleurotus ostreatus strains for commercial cultivation under warm environmental conditions. Scientia Horticulturae 118(2): 106-110.

Geetha, D., K. Sivaprakasam, 1993. Pleurotus-djamor - a new edible mushroom. Current Science 64(5): 280-281.

Guo, L.Q., J.Y. Lin, J.F. Lin, 2007. Non-volatile components of several novel species of edible fungi in China. Food Chemistry 100(2): 643-649.

Guzmán, G., 2000. Genus Pleurotus (Jacq.: Fr.) P. Kumm. (Agaricomycetideae): diversity, taxonomic problems, and cultural and traditional medicinal uses. International Journal of Medicinal Mushrooms 2: 95-123.

Guzmán, G., L. Montoya, V.M. Bandala, G. Mata, D. Salmones, 1995. Studies in the genus Pleurotus, IV. Observations on the pink forms growing in México based in the interbreeding of two different strains. Mycotaxon 53: 247-259.

Guzmán, G., L. Montoya, D. Salmones, V.M. Bandala, 1993. Studies on the genus Pleurotus (Badidiomycotina), II. P. djamour in Mexico an in other Latin-american countries, taxonomic confusions, distribution and semi-industrial culture. Cryptogamic Botany 3(2): 213-220.

Guzmán, G., M. Piepenbring, 2011. Los hongos de Panamá: introducción a la identificación de los macroscópicos. Instituto de Ecología, A. C., Xalapa.

Guzmán, M., N. Zúñiga, G.G. Santafé, O. Torres, A. Angulo, 2009. Actividad antioxidante y estudio químico del hongo Pleurotus djamor recolectado en Córdoba. Facultad de Ciencias Agropecuarias 7(2): 63-60.

Hasan, M.T., M.H.A. Khatun, M.A.M. Sajib, M.M. Rahman, M.S. Rahman, M. Roy, M.N. Miah, K.U. Ahmed, 2015. Effect of wheat bran supplement with sugarcane bagasse on growth, yield and proximate composition of pink oyster mushroom (Pleurotus djamor). American Journal of Food Science and Technology 3(6): 150-157.

Hernández-Ibarra, H., J.E. Sánchez-Vázquez, L.A. Calvo Bado, 1995. Estudio de 5 cepas nativas de Pleurotus spp. de la region de Tapachula, Chiapas, México. Revista Mexicana de Micología 11: 29-83.

Ho, J.C.K., S.C.W. Sze, W.Z. Shen, W.K. Liu, 2004. Mitogenic activity of edible mushroom lectins. Bioquimica et Biophysica Acta 1671(1-3): 9-17.

Huerta, G., D. Martínez-Carrera, J.E. Sánchez Vázquez, H. Leal Lara (2009) Grupos de interesterilidad y productividad de cepas de Pleurotus de regiones tropicales y subtropicales de México. Revista Mexicana de Micología 30: 31-42.
Iracabal, B., G. Zervakis, J. Labarere, 1995. Molecular systematics of the genus Pleurotus - analysis of restriction polymorphisms in ribosomal DNA. Microbiology 141 (6):1479-1490.

James, T.Y., S.R. Liou, R. Vilgalys, 2004. The genetic structure and diversity of the A and B mating-type genes from the tropical oyster mushroom, Pleurotus djamor. Fungal Genetics and Biology 41(8): 813-825.

Kalmis, E., N. Azbar, F. Kalvoncu, 2007. Agar-plate screening for textile dye decolorisation by white rot fungi Pleurotus species (Pleurotus cornucopiae var.citrino-pileatus, P-djamor, P-eryngii, P-ostreatus and P-sajor-caju). Fresenius Environmental Bulletin 16(10): 1309-1314.

Kalmis, E., N. Azbar, F. Kalvoncu, 2008. Evaluation of two wild types of Pleurotus ostreatus (MCC07 and MCC20) isolated from nature for their ability to decolorize Benazol Black ZN textile dye in comparison to some commercial types of white rot fungi: Pleurotus ostreatus, Pleurotus djamor, and Pleurotus citrinopileatus. Canadian Journal of Microbiology 54(5): 366-370.

Khan, M.A., T. Mousumi, 2012. Nutritional and medicinal Importance of Pleurotus mushrooms: an overview. Food Review International 28(3): 313-329.

Khan, N. A., M. Ajmal, J. Nicklin, S. Aslam, M.A. Ali, 2013. Nutritional value of Pleurotus flabellatus djamor (R-22) cultivated on sawdust of different woods. Pakistan Journal of Botany 45(3): 1105-1108.

Kidukuli, A.W., Z.H. Mbwambo, H. Malebo, C.A. Mgina, M.J. Mihale, 2010. In vivo antiviral activity, protease inhibition and brine shrimp lethality of selected Tanzanian wild edible mushrooms. Journal Applied of Bioscience 31: 1887-1894.

Kivaisi A.K., F.S.S. Magingo, B. Mamiro, 2003. Performance of Pleurotus flabellatus on water hyacinth (Eichbornia crassipes) shoots at two different temperature and relative humidity regimes. Tanzania Journal Science 29(2): 11-18.

Lechner, E.B., J.E. Wright, 2004. The genus Pleurotus in Argentina. Mycologia 96(4): 845-858.

Legon, N.W., 1999. A mycological expedition to Puerto Rico. Mycologist 13(2): 58-62.

Machado, K.M.G., D.R. Matheus, V.L.R. Bononi, 2005. Ligninolytic enzymes production and remazol brilliant blue $\mathrm{R}$ decolorization by tropical brazilian basidiomycetes fungi. Brazilian Journal of Microbiology 36: 246-252.

Leonowicz, A., N.S. Cho, J. Luterek, A. Wilkolazka, M. Wojtas-Wasilewska, A. Matuszewska, M. Hofrichter, D. Wesenberg, J. Rogalski, 2001. Fungal laccase: properties and activity on lignin. Journal of Basic Microbiology 41: 185-22.

Martínez-Carrera, D., A. Larqué-Saavedra, A. Tovar Palacio, N. Torres, M.E. Meneses, M. Sobal Cruz, P. Morales Almora, M. Bonilla Quintero, H. Escudero Uribe, I. Tello-Salgado, T. Bernabé-González, W. Martínez Sánchez, Y. Mayett, 2016. Contribución de los hongos comestibles, funcionales y medicinales a la construcción de un paradigma sobre la producción, la dieta, la salud y la cultura en el sistema agroalimentario de México. In: Martínez-Carrera, D., J. Ramírez Juárez (eds.), Ciencia, tecnología, innovación en el sistema agroalimentario de México. COLpos-amc-Conacyt-upaep-iminap. Texcoco. Pp. 581-640.

Mata, G., E. González, D. Salmones, 2007. Micelial growth of three Pleurotus (Jacq.:Fr.) P. Kumm. species on sugarcane bagasse: production of hydrolytic and oxidative enzymes. International Journal of Medicinal Mushrooms 9: 385-394.

Mata, G., D.M. Murrieta Hernández, L.G. Iglesias Andreu, 2005. Changes in lignocellulolytic enzyme activites in six Pleurotus spp. strains cultivated on coffee pulp in confrontation with Trichoderma spp. World Journal of Microbiology and Biotechnology 21: 143-150.

Mata, G., D. Salmones, J.M. Savoie, 2017. Enzimas lignocelulíticas de Pleurotus spp. In: Sánchez, J.E., D. Royse (eds.), La biología, el 
cultivo y las propiedades nutricionales y medicinales de las setas Pleurotus spp. El Colegio de la Frontera Sur. Tapachula. Pp. 63-82.

Melgarejo, E. 2015. Algunos usos de los hongos silvestres de Bolivia en el contexto sudamericano. Kempffiana 11(1): 48-65.

Menolli Junior, N., T. Asai, M. Capeari, L. D. Paccola-Meirelles, 2010. Morphological and molecular identification of four Brazilian commercial isolates of Pleurotus spp. and cultivation on corncob. Brazilian Archives of Biology and Technology 53(2): 397408.

Menolli, N., B.S. Breternitz, M. Capelari, 2014. The genus Pleurotus in Brazil: a molecular and taxonomic overview. Mycoscience 55(5): 378-389.

Miles, P.G., S.T. Chang, 2004. Mushrooms: cultivation, nutritional value, medicinal effect, and environmental impact. CRC Press, Hong Kong.

Milton, R., R. Dulay, L.A. Miranda, J.S. Malasaga Sofronio, P. Kalaw, R.G. Reyes, C.T. Hou, 2017. Antioxidant and antibacterial activities of acetonitrile and hexane extracts of Lentinus tigrinus and Pleurotus djamour. Biocatalysis and Agricultural Biotechonology 9: 141-144.

Morales, O., M. Bran, R. Cáceres, R. Flores, 2003. Contribución al conocimiento de los hongos comestibles de Guatemala. Documento interno, Facultad de Ciencias Químicas y de Farmacia, Universidad de San Carlos, Guatemala.

Motato, R., I.A. Mejía, A. León, 2006. Evaluación de los residuos agroindustriales de plátano (Musa paradisíaca) y aserrín de abarco (Cariniana piriformes) como sustratos para el cultivo del hongo Pleurotus djamor. Vitae 13:24-29.

Mshandete, A.M., J. Cuff, 2007. Proximate and nutrient composition of three types of indigenous edible wild mushrooms grown in Tanzania and their utilization prospects. African Journal of Food, Agriculture, Nutrition and Development 7(6): 1-16.

Mshandete, A.M., J. Cuff, 2008. Cultivation of three types of indigenous wild edible mushrooms: Coprinus cinereus, Pleurotus flabellatus and Volvariella volvocea on composted sisal decortications residue in Tanzania. African Journal of Biotechnology 7(24): 4551-4562.

Murakami, S., T. Takemaru, 1990. Genetic studies of Pleurotus salmoneostramineus forming albino basidiocarps. Reports of the Tottori Mycological Institute 28: 199-204.

Neda, H., H. Furukawa,T. Miyagi, 1988. Two Pleurotus species from Okinawa. Proc. 32nd Annual Meeting Mycological Society of Japan. P. 51.

Nicholl, D.B.G., R.H. Petersen, 2000. Phenetic plasticity in Pleurotus djamor. Mycotaxon 76: 17-37.

Nilsson, I., Möller, A., Mattiasson, B., Rubindamayugi, M.S.T., U. Welander., 2006. Enzyme and microbial technology decolorization of synthetic and real textile wastewater by the use of whiterot fungi. Enzyme and Microbial Technology 38 (1-2): 94-100.

Okano, K., Y. Iida, M. Samsuri, B. Prasetya, T. Wsagawa, T. Watanabe, 2006. Comparison of in vitro digestibility and chemical composition among sugarcane bagasses treated by four whiterot fungi. Animal Science Journal 77(3): 308-313.

Paswall, S., N. Mughal, V. Bharti, S. Mahajan, M. Majeed, 2017. Evaluation of locally available substrates for sporocarps production of pink Pleurotus [Pleurotus djamor (Rumph.ex.Fr.) Boedijn] mushroom. International Journal of Current Microbiology and Applied Sciences 6(12): 1677-1684.

Pegler, D.N., 1977. A preliminary agaric flora of east Africa. Kew Bulletin Additional Series 6. Kew.

Pegler, D.N., 1983. Agaric flora of the Lesser Antilles. Kew Bulletin Additional Series 9. Kew.

Pegler, D.N., 1986. Agaric flora of Sri Lanka. Kew Bulletin Aditional Series 12. Kew.
Petersen, R.H., K.W. Hughes, 1993. Intercontinental interbreeding collections of Pleurotus pulmonarius, with notes on P. ostreatus and other species. Sydowia 45: 139-152.

Petersen, R.H., G.S. Ridley, 1996. A New Zealand Pleurotus with multiple-species sexual compatibility. Mycologia 88: 198-207.

Pineda-Alegría, J.A., J.E. Sánchez-Vázquez, M. González-Cortazar, A. Zamilpa, M.E. López-Arellano, E.J. Cuevas-Padilla, P. Mendoza de Gives, L. Aguilar-Marcelino, 2017. The edible mushroom Pleurotus djamor produces metabolites with lethal activity against the parasitic nematode Haemonchus contortus. Journal of Medicinal Food 20(12): 1-9.

Putzke, J., 2002. Los géneros Pleurotus e Lentinus (Agaricales, Basidiomycota, Fungos) no Brasil1. Lista de especies e chaves de identificacão. Caderno de Pesquisa Series Biologia (Santa Cruz do Sul) $14: 67-75$.

Quimio J.H., 1978 Introducing Pleurotus flabellatus for your dinner table. Mushroom Journal 68: 282-283.

Rajarathnam S., Z. Bano, M.V. Patwardhan, 1986. Nutrition of the mushroom Pleurotus flabellatus during its growth on paddy straw substrate. Journal Horticulture Science 61(2): 223-232.

Rajarathnam S., D.B. Wankhede, M.V. Patwardhan, 1979. Some chemical and biochemical changes in straw constituents during growth of Pleurotus flabellatus (Berk \& Br) Sacc. European Journal of Applied Microbiology and Biotechnology 8: 125-134.

Rajarathnam S., D.B. Wankhede, Z. Bano, 1987. Degradation of rice straw by Pleurotus flabellatus. Journal of Chemical Technology and Biotechnology 37(3): 203-214.

Rajarathnam, S., M. Nanjaraja, U. Shashirekha, Z. Bano, 1998. Biodegradative and biosynthetic capacities of mushrooms: present and future strategies. Critical Reviews in Biotechnology 18 (2-3): 91-236.

Rampinelli J.R., Silveira M.L.L., Gern, M.R.R., Furlan, S.A., Ninow J.L., Wisbeck, E. 2010. Nutritional value of Pleurotus djamor cultivated on banana straw. Alimentos e Nutricao 21(2): $197-$ 202.

Ranzani, L.R.T.D., G.L. Sturion, 1998. Amino acid composition evaluation of edible mushrooms (Pleurotus spp.) cultivated on banana leaves. Archivos Latinoamericanos de Nutrición 48(4): 339-348.

Rodrigues Alexandre, T., M. Lopes Lima, M. Kolos Galupp, J. Tonini Mesquita, M.A. do Nascimento, A. L. dos Santo, P. Sartorelli, D. Carvalho Pimenta, A.G, Tempone, 2017. Ergosterol isolated from the basidiomycete Pleurotus salmoneostramineus affects Trypanosoma cruzi plasma membrane and mitochondria. Journal of Venomous Animals and Toxins including Tropical Diseases 23: 30.

Rolz, C.R. de León, M.C. de Arriola, S. de Cabrera, 1986. Biodelignification of lemon grass and citronella bagasse by white-rot fungi. Applied and Environmental Microbiology 52(4): 607-611.

Rosa, L.H., K.M.G. Machado, A.L.T. Rabello, E.M. Souza-Fagundes, R. Correa-Oliveira, C.A. Rosa, C.L. Zani, 2009. Cytotoxic, immunosuppressive, trypanocidal and antileishmanial activities of Basidiomycota fungi present in Atlantic Rainforest in Brazil. Antonie Van Leeuwenhoek International Journal of General and Molecular Microbiology 95(3): 227-237.

Rosado, F.R., E.R. Carbonero, C. Kemmelmeier, C.A. Tischer, P.A.J. Gorin, M. Iacomini, 2002a. A partially 3-0-methylated (1-4)-linked $\alpha$-D-galactan and $\alpha$-D-mannan from Pleurotus ostreatorroseus Singer. FEMS Microbiology Letters 212(2): 261265.

Rosado, F.R., C. Kemmerlmeier, S.M. Gomes Da Costa, 2002b. Alternative method of inoculum and spawn production for the cultivation of the edible Brazilian mushroom Pleurotus ostreatoroseus Sing. Journal of Basic Microbiology 42(1):37-44.

Royse, D.J., J.E. Sánchez, 2017. Producción mundial de setas Pleurotus spp. con énfasis en países iberoamericanos. In: Sánchez, J.E., D. 
Royse (eds.). La biología, el cultivo y las propiedades nutricionales y medicinales de las setas Pleurotus spp. El Colegio de la Frontera Sur. Tapachula, pp. 17-25.

Royse, D.J., S.A. Zaki, 1991. Yield stimulation of Pleurotus-flabellatus by dual nutrient supplementation of pasteurized wheat straw. Mushroom Science 13(1-2): 545-547.

Royse, D.J., J. Baars, Q. Tan, 2017. Current overview of mushroom production in the world. In: Zied, D.C., A. Pardo-Giménez (eds.), Edible and medicinal mushrooms: Technology and applications. John Wiley and Sons-Blackwell, Nueva York. Pp. 5-13.

Salmones, D., R. Gaitán-Hernández, R. Pérez, G. Guzmán, 1997. Estudios sobre el género Pleurotus VIII. Interacción entre crecimiento micelial y productividad. Revista Iberoamericana de Micología 14: 173-176.

Salmones, D., G. Mata, 2002. Detection of extracellular enzymes produced by Pleurotus spp. grown on coffee pulp. In: Sánchez, J.E., G. Huerta, E. Montiel (eds.), Mushroom Biology and Mushroom Products, proc. $4^{\text {th }}$ International Conference. Universidad Autónoma de Morelos. Cuernavaca. Pp. 213-220.

Salmones, D., G. Mata, 2015. Laccase production by Pleurotus djamor in agar media and during cultivation on wheat straw. Revista Mexicana de Micología 42: 17-23.

Salmones, D., G. Mata, 2017. Recursos genéticos del género Pleurotus. In: Sánchez, J.E., D. Royse (eds.) La biología, el cultivo y las propiedades nutricionales y medicinales de las setas Pleurotus spp. El Colegio de la Frontera Sur. Tapachula. Pp. 29-52.

Salmones, D., G. Mata, G. Guzmán, M. Juárez, L. Montoya, 1995. Estudios sobre el género Pleurotus, V. Producción a nivel de planta piloto de ocho cepas adscritas a cinco taxa. Revista Iberoamericana de Micología. 12: 108-110.

Salmones, D., G. Mata, K. Waliszewski, 2005. Comparative culturing of Pleurotus spp. on coffee pulp and wheat straw: biomass production and substrate degradation. Bioresource Technoogy 96: 537-544.

Salmones, D., L. Mestizo Valdéz, R. Gaitán-Hernández, 2004. Entrecruzamiento y evaluación de la producción de las variedades de Pleurotus djamor (Fr.) Boedijn. Revista Mexicana de Micología 18: 21-26.

Sánchez, J.E., G.M. Orozco, D. Hernández Rodríguez, M.G. Nieto, F.J. Márquez, 2007. El sustrato degradado por Pleurotus pulmonarius para la degradación del insecticida endosulfán. In: Sánchez Vázquez, J.E., D. Martínez Carrera, G. Mata, H. Leal Lara (eds.), El cultivo de setas Pleurotus spp. en México. El Colegio de la Frontera Sur, Tapachula. Pp. 199-207.

Sánchez-Cantú, M., L. Ortiz-Moreno, M.E. Ramos Cassellis, M. Marín-Castro, C. de la Cerna-Hernández, 2017. Solid-state treatment of castor cake employing the enzymatic cocktail produced from Pleurotus djamor fungi. Applied Biochemistry and Biotechnology 1-16. https://doi.org/10.1007/s12010-0172656-4

Santos, A.Z., C.R.G. Tavares, S.M. Gomes-da-Costa, 2002. Treatment of the effluent from a kraft bleach plant with the white-rot fungus Pleurotus ostreatoroseus Sing. Brazilian Journal of Chemical Engineering 19(4): 371-375.

Savoie, J.M., D. Salmones, G. Mata, 2007. Hydrogen peroxide concentration measured in cultivation substrates during growth and fruiting of mushrooms, Agaricus bisporus and Pleurotus spp. Journal of Science Food and Agriculture 87: 1337-1344.

Selvakumar, P., S. Rajasekar, A.M Babu, K. Periasamy, N. Raaman, M. Sudhakara Reddy, 2015. Improving biological efficiency of Pleurotus strain through protoplast fusion between $P$. ostreatus var. florida and P. djamor var. roseus. Food Science and Biotechno$\log$ 24(5): 1741-1748.

Selvakumar, G., S. Saha, S. Kundu, 2007. Inhibitory activity of pine needle tannin extracts on some agriculturally resourceful microbes. Indian Journal of Microbiology 47: 267-270.
Sudha, G., A. Janardhanan, A. Moorthy, M. Chinnasamy, S. Gunasekaran, A. Thimmaraju, J. Gopalan, 2016. Comparative study on the antioxidant activity of methanolic and aqueous extracts from fruiting bodies of an edible mushroom Pleurotus djamor. Food Science and Biotechnology 25(2): 371-377.

Suresh, N., J. Ambika, A. Noorjahan, M. Kalaiselvam, 2017. Pink oyster mushroom (Pleurotus djamor) and its efficacy against human pathogen. International Journal of Science Innovations Today 6(6): 749-757.

Thakur, S., M.S. Chauhan, 2018. Treatment of dye wastewater from textile industry by electrocoagulation and Fenton oxidation: A review. In: Singh, V., S. Yadav, R. Yadava (eds.), Water Quality Management. Water Science and Technology Library, vol. 79. Springer, Singapur. Pp. 117-129.

Upadhyay, R.C, M. Hofrichter, 1993. Effect of phenol on the mycelial growth and fructification in some basidiomycetous fungi. Journal of Basic Microbiology 33(5): 343-347.

Valencia del Toro, G., M.E. Garín Aguilar, J. Jiménez Hernández, H. Leal-Lara, 2003. Producción de cepas coloridas de Pleurotus spp. en sustrato estéril y pasteurizado. Revista Mexicana de Micología 17: 1-5.

Valencia del Toro, G., H. Leal-Lara, 1999. Estudios de compatibilidad entre cepas de Pleurotus spp. con cuerpos fructíferos de diferentes colores. Revista Mexicana de Micología 15: 65-71.

Valencia del Toro, G., M.E. Garín Aguilar, M.A. Téllez Jiménez, E. Durán Páramo, 2008. Actividad antibacteriana de extractos hexánicos de cepas de Pleurotus djamor. Revista Mexicana de Micología 28: 119-123.

Vega, A., G. Mata, D. Salmones, R.E. Caballero, 2006. Cultivo de cepas nativas de Pleurotus djamor en Panamá, en paja de arroz y pulpa de café. Revista Mexicana de Micología 23: 93-97.

Vega, A., H. Franco, 2013. Productividad y calidad de los cuerpos fructíferos de los hongos comestibles Pleurotus pulmonarius RN2 y $P$. djamor RN81 y RN82 cultivados sobre sustratos lignocelulósicos. Información Tecnológica 24(1): 69-78.

Villers-Ruiz, L., I. Trejo-Vázquez, 1998. Impacto del cambio climático en los bosques y áreas naturales protegidas de México. Interciencia 23(1): 10-19.

Wu, X., S. Zheng, L. Cui, H. Wang, T.B. Ng, 2010. Isolation and characterization of a novel ribonuclease from the pink oyster mushroom Pleurotus djamor. Journal of Genetics and Applied Microbiology 56(3): 231-239.

Yoon, K.N., N. Alam, M.J. Shim, T.S. Lee, 2012. Hypolipidemic and antiatherogenesis effect of culinary-medicinal pink oyster mushroom, Pleurotus salmoneostramineus L. Vass. (higher Basidiomycetes) in hypercholesterolemic rats. International Journal of Medicinal Mushroms 14(1): 27-36.

Zawirska-Wojtasiak, R., M. Siwulski, S. Mildner-Szkudlarz, E. Wasowicz, 2009. Studies on the aroma of different species and strains of Pleurotus measured by GC/MS, sensory analysis and electronic nose. Acta Scientiarum Polonorum Technologia Alimentaria 8(1): 47-61.

Zervakis, G., J. Labarere, 1992. Taxonomic relationships within the fungal genus Pleurotus as determined by isoelectric-focusing analysis of enzyme patterns. Journal of General Microbiology 138: 635-645.

Zervakis, G., J. Sourdis, C. Balis, 1994. Genetic-variability and systematics of 11 Pleurotus species based on isozyme analysis. Mycological Research 98(3): 329-341.

Zhang, J., M. Liu, Y.H. Yang, L. Lin, N. Xu, H.J. Zhao, L. Jia, 2016. Purification, characterization and hepatoprotective activities of mycelia zinc polysaccharides by Pleurotus djamor. Carbohydate Polymers 136: 588-597.

Zhang, J., G. Meng, C. Zhang, N. Lin, N. Xu, M. Liu, F. Cui, L. Jia, 2015. The antioxidative effects of acidic-, alkalic-, and enzymatic-extractable mycelium zinc polysaccharides by Pleurotus dja- 